Supplementary materials.

Noureddine Dadda, Nassour Ayoub, Benoît Guillot, Benalicherif Nourredine \& Christian Jelsch*

Charge-density analysis and electrostatic properties of 2-Carboxy-4-methylanilinium chloride monohydrate obtained using a multipolar model and a spherical charges model.

Table S1. Wave function coefficients and orbital exponents for the virtual atom described as a combination of Slater functions.

$\operatorname{Slater}\left(N_{1}, \xi, r\right)=\operatorname{coeff} * r^{N 1} * \exp (-\xi r)$

\begin{tabular}{|l|l|l|}
\hline ATOM & $\xi$ & Coeff. \\
\hline 0 & 6.26445 & 0.07360 \\
\hline 1 & 6.06204 & 0.13954 \\
\hline 2 & 3.73854 & 0.87683 \\
\hline
\end{tabular}


Figure S1. Analysis of experimental vs. expected $\Delta \rho$.

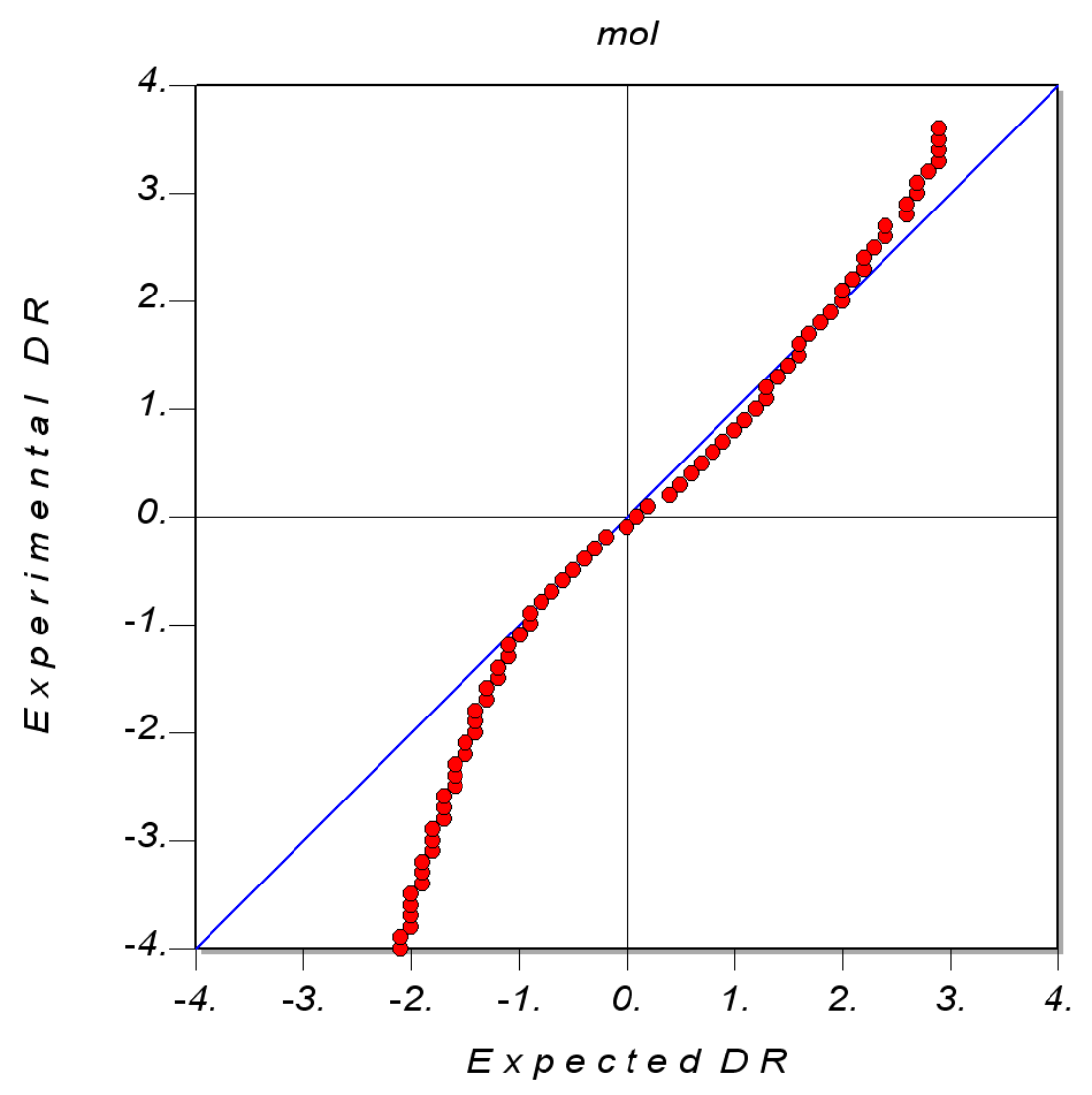

Figure S2. Analysis of $\left\langle F_{\mathrm{O}}\right\rangle \mid\left\langle F_{\mathrm{c}}\right\rangle$ as a function of reciprocal resolution.

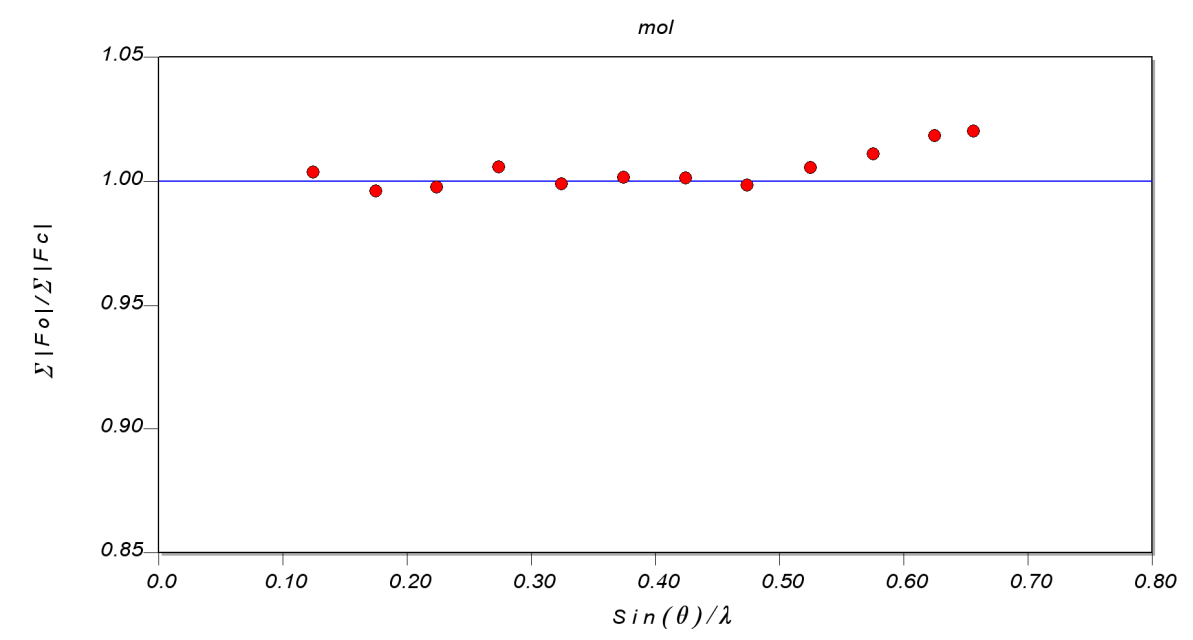




\section{Figure S3.}

Experimental residual electron density in the lone pairs planes.

Contour level: $\pm 0.05 \mathrm{e} / \AA^{3}$. Blue solid lines and red dashed lines denote positive and negative contours, respectively. The zero contours is shown as yellow line.
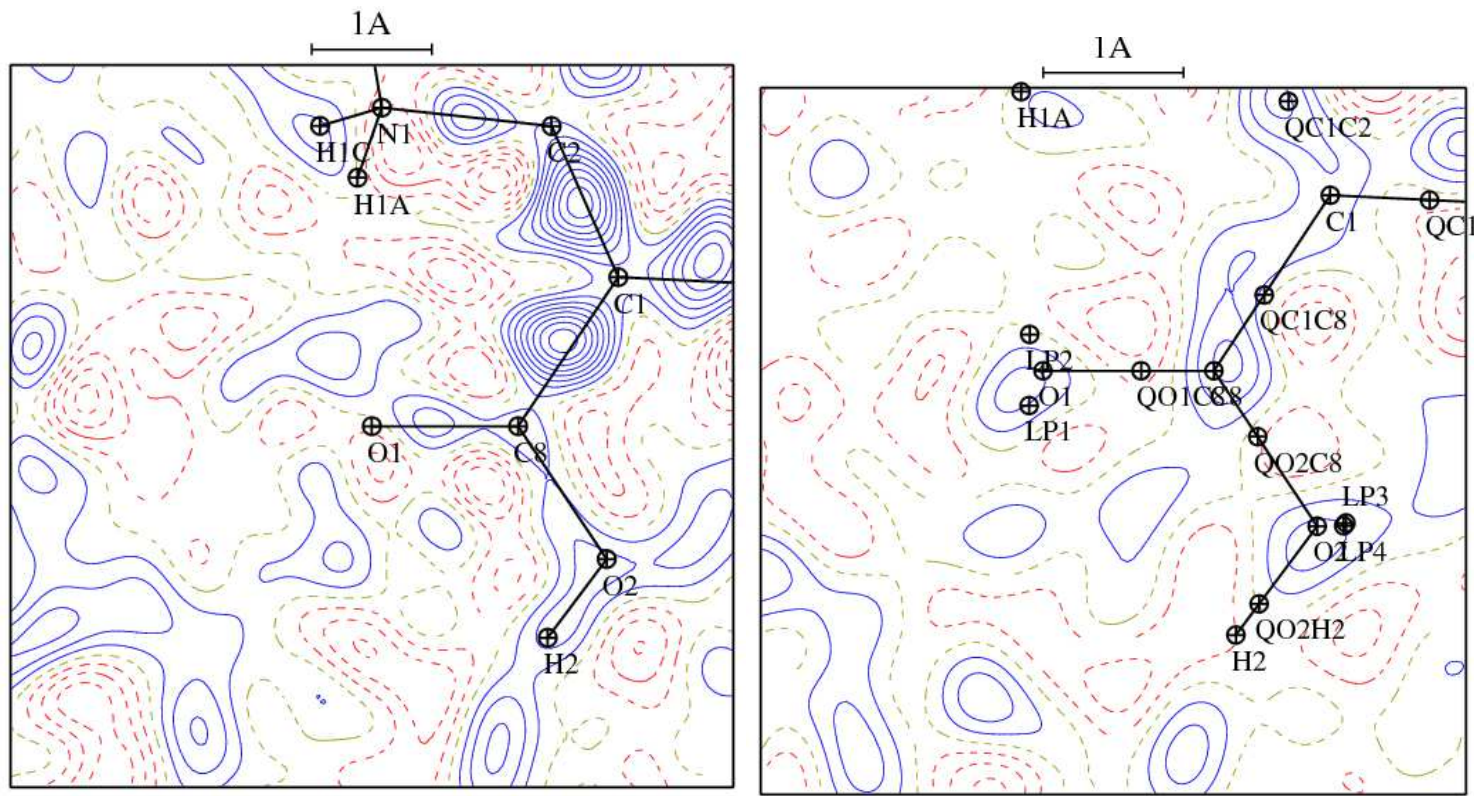

(a) EXP_IAM

(b) EXP_VIR

in the $\mathrm{C} 8 \mathrm{O} 1 \mathrm{O} 2$ plane
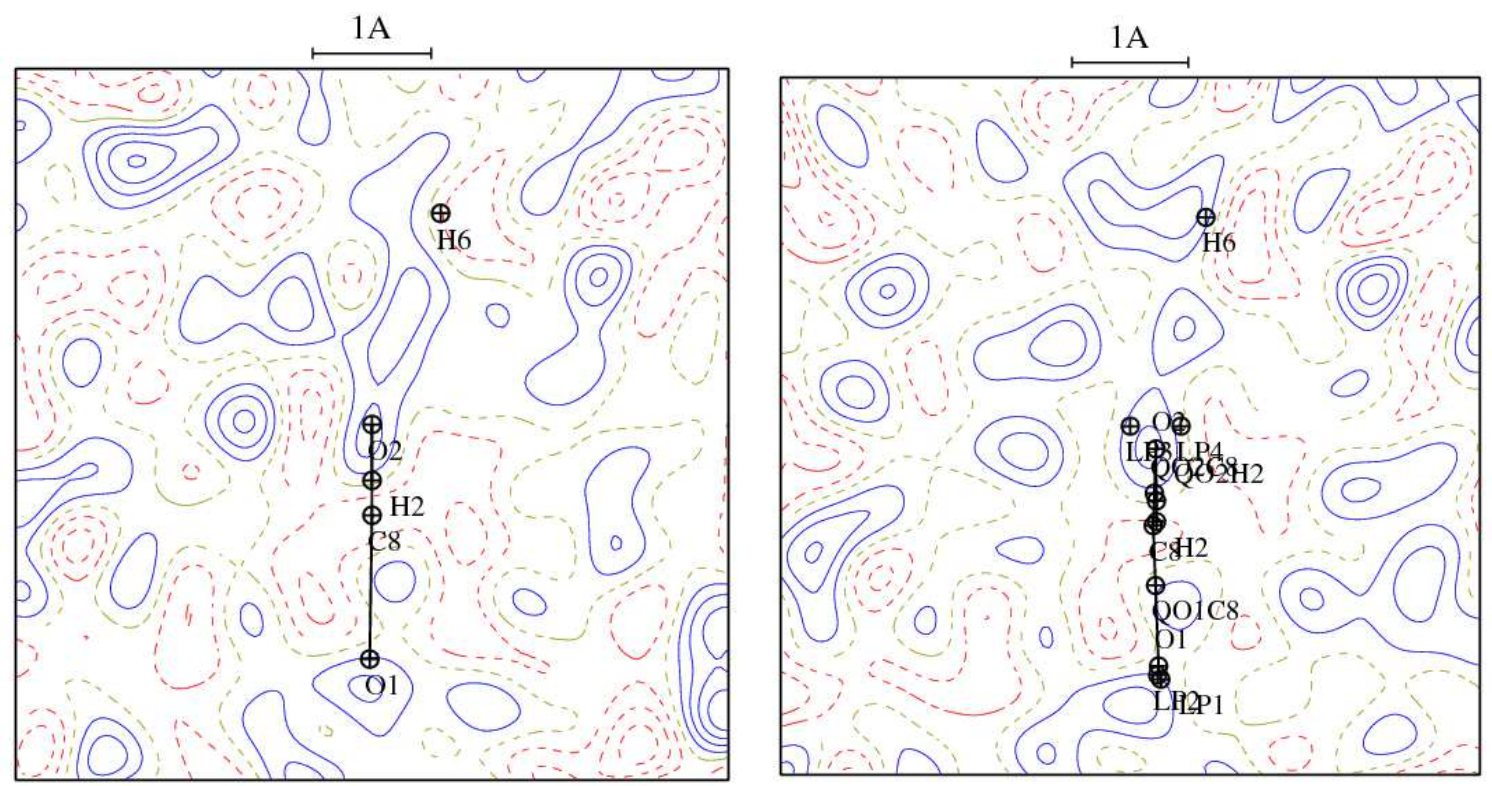

(c) EXP_IAM

(d) EXP_VIR

in the oxygen atom $\mathrm{O} 2$ lone pairs plane. 


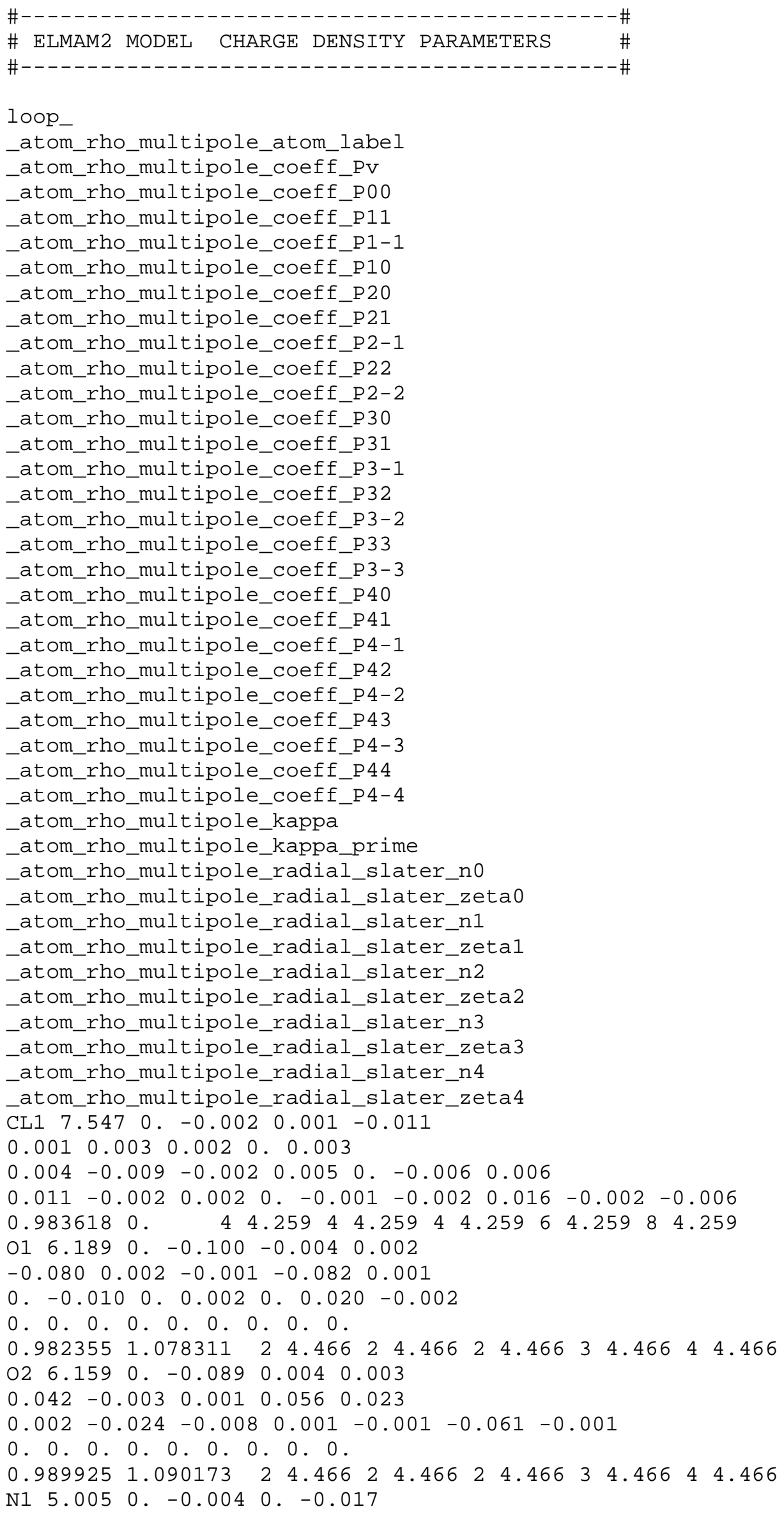




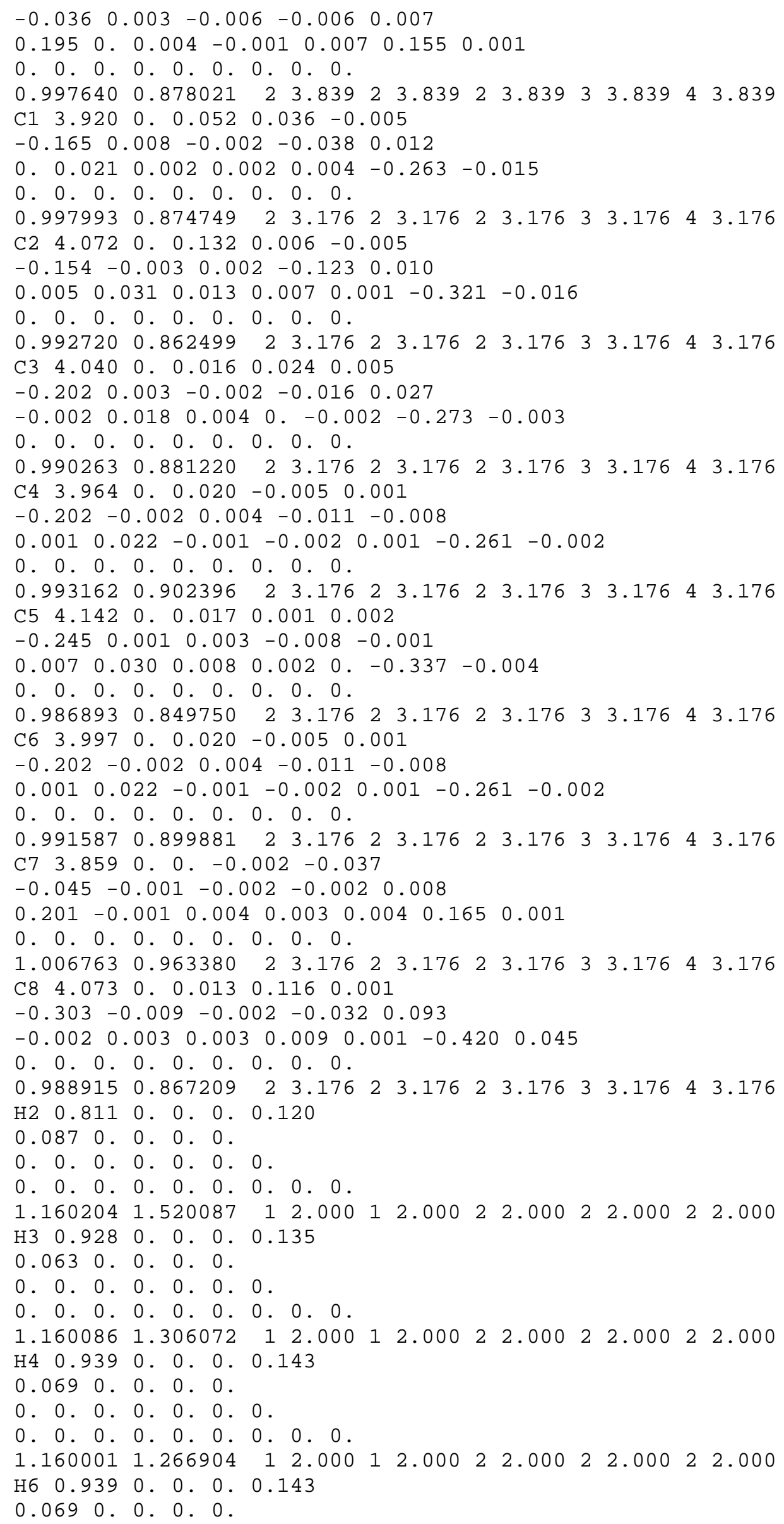




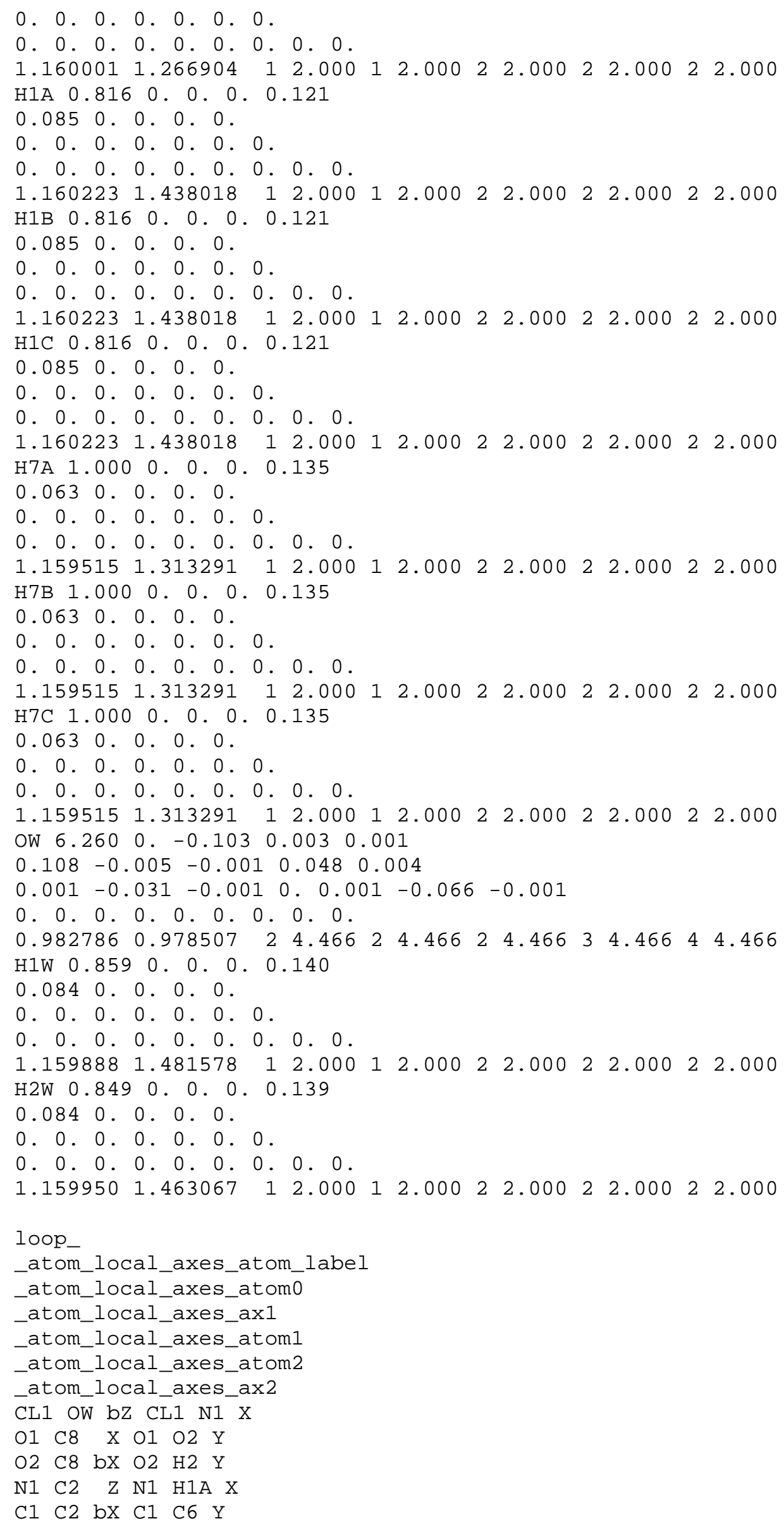




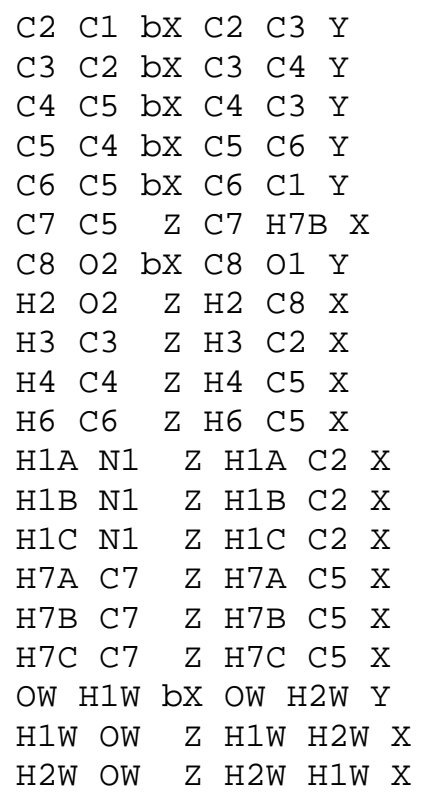

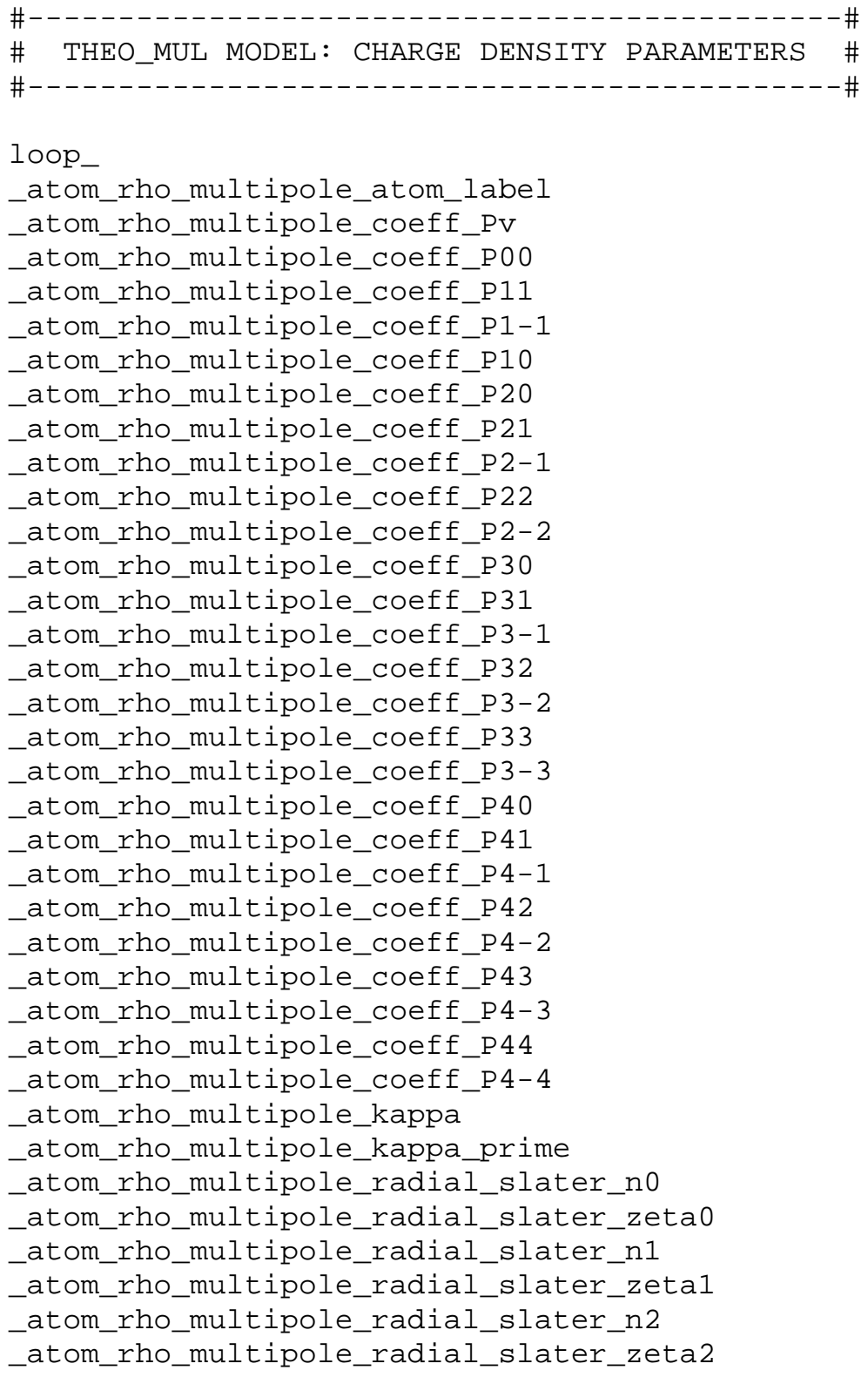




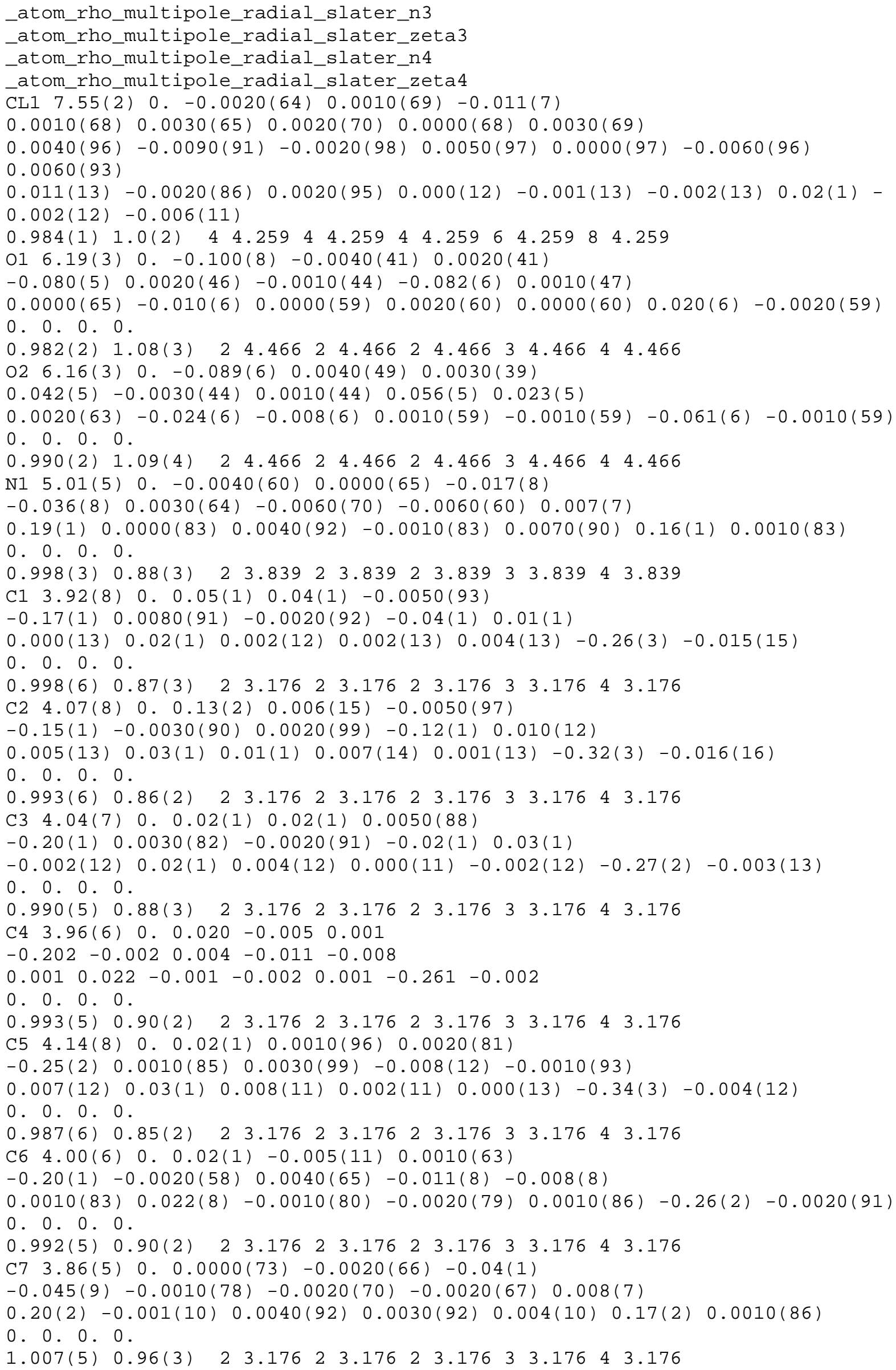


C8 $4.07(6) \quad 0.0 .01(1) \quad 0.12(1) \quad 0.0010(84)$

$-0.30(2)-0.009(9)-0.0020(83)-0.03(1) \quad 0.09(1)$

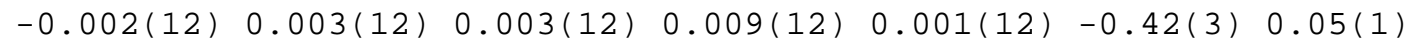

0.0 .0 .0 .

$\begin{array}{llllllllllllllll}0.989(5) & 0.87(2) & 2 & 3.176 & 2 & 3.176 & 2 & 3.176 & 3 & 3.176 & 4 & 3.176\end{array}$

$\mathrm{H} 20.81(1) 0.0 .0 .0 .12(1)$

$0.09(1) 0.0 .0 .0$.

0 . 0 .

0.0 .0 .0 .

$1.160(2) 1.52(8) \quad 12.00012 .00022 .00022 .00022 .000$

H3 $0.93(2) 0.0 .0 .0 .135(7)$

$0.063(6) 0.0 .0 .0$.

0.0 .

0.0 .0 .0 .

$1.160(2) 1.31(5) \quad 12.00012 .00022 .00022 .00022 .000$

$\mathrm{H} 4 \quad 0.9390 .0 .0 .0 .143$

0.0690 .0 .0 .0 .

0.0 .

0.0 .0 .0 .

$1.160(2) 1.27(5) \quad 12.00012 .00022 .00022 .00022 .000$

H6 $0.94(1) \quad 0.0 .0 .0 .14(1)$

$0.069(10) 0.0 .0 .0$.

0 . 0 .

0.0 .0 .0 .

$1.160(2) 1.27(5) \quad 12.00012 .000 \quad 22.00022 .00022 .000$

$\mathrm{H} 1 \mathrm{~A} \quad 0.82(1) \quad 0.0 .0 .0 .121(7)$

$0.085(7) 0.0 .0 .0$.

0 . 0 .

0.0 .0 .0 .

$1.160(2) 1.44(4) \quad 12.00012 .000 \quad 22.000222 .00022 .000$

$\mathrm{H} 1 \mathrm{~B} \quad 0.8160 .0 .0 .0 .121$

0.0850 .0 .0 .0 .

0.0 .

0.0 .0 .0 .

$1.160(2) 1.44(4) \quad 12.00012 .000 \quad 22.000222 .000222 .000$

$\mathrm{H} 1 \mathrm{C} 0.8160 .0 .0 .0 .121$

0.0850 .0 .0 .0 .

0 . 0 .

0.0 .0 .0 .

$1.160(2) 1.44(4) \quad 12.00012 .00022 .00022 .00022 .000$

H7A $1.00(1) 0.0 .0 .0 .135$

0.0630 .0 .0 .0 .

0.0 .

0.0 .0 .0 .

$1.160(2) 1.31(4) \quad 12.00012 .000222 .000222 .000222 .000$

H7B 1.000 0.0.0.0.135

0.0630 .0 .0 .0 .

0.0 .

0.0 .0 .0 .

$1.160(2) 1.31(4) \quad 12.00012 .000 \quad 22.000222 .000222 .000$

$\mathrm{H} 7 \mathrm{C} 1.0000 .0 .0 .0 .135$

0.0630 .0 .0 .0 .

0.0 .

0.0 .0 .0 .

$1.160(2) 1.31(4) \quad 12.00012 .000 \quad 22.000222 .000222 .000$

OW $6.26(3) \quad 0 .-0.103(8) \quad 0.0030(60) \quad 0.0010(41)$

$0.108(7)-0.005(5)-0.0010(46) \quad 0.048(5) \quad 0.0040(58)$

$0.0010(67)-0.031(7)-0.0010(65) \quad 0.0000(64) \quad 0.0010(64)-0.066(7) \quad-$

$0.0010(64)$

0.0 .0 .0 . 0.

$0.983(2) \quad 0.98(3) \quad 24.46624 .46624 .466 \quad 3 \quad 4.466 \quad 4 \quad 4.466$ 


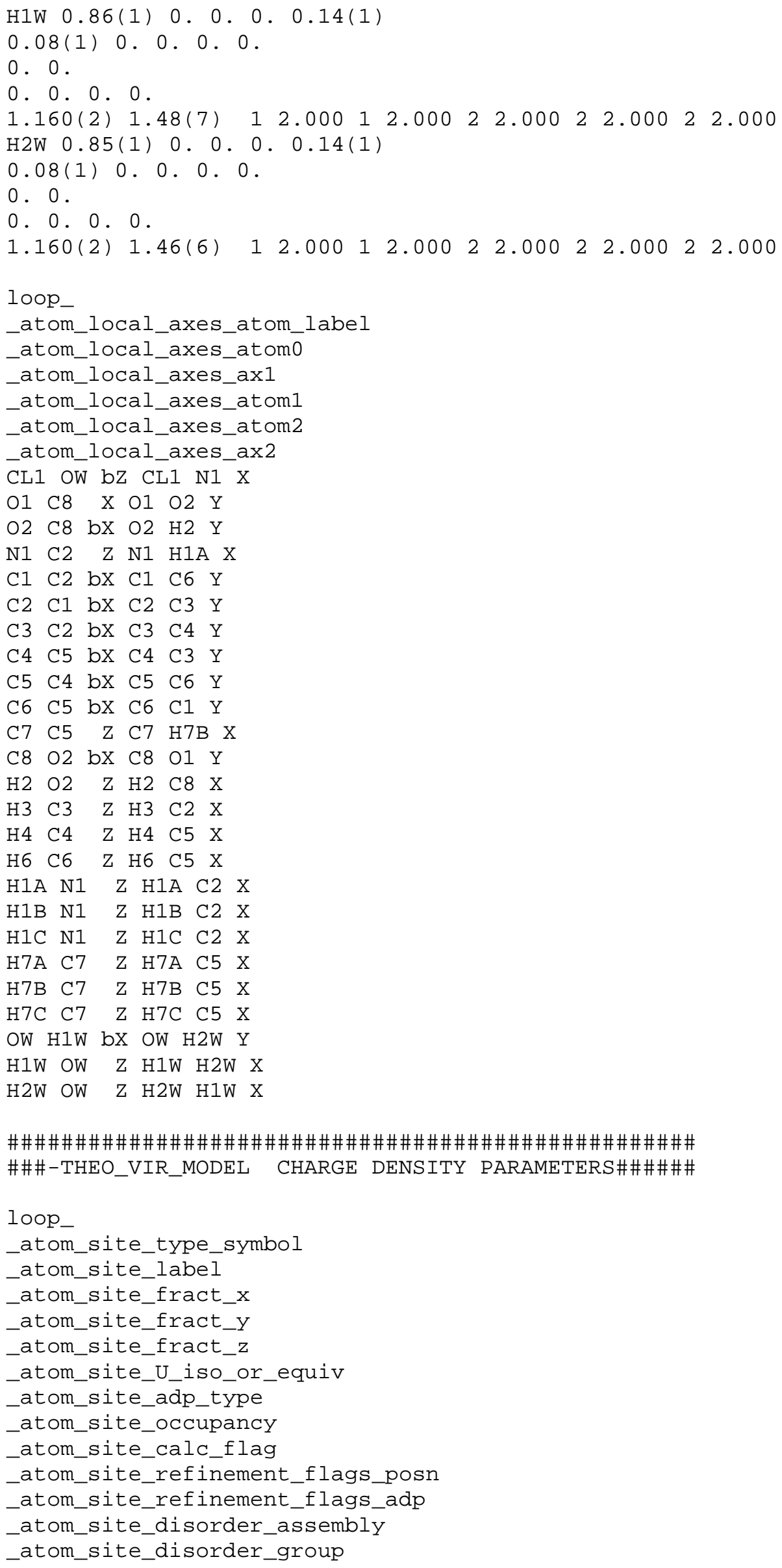


C1 CL1 $0.16000 \quad 0.49786 \quad 0.677120$.

$\begin{array}{llllllll}0 & 01 & 0.17413 & 0.46924 & 0.30185 & 0 .\end{array}$.

$\begin{array}{lllllll}0 & 02 & 0.37352 & 0.52449 & 0.36549 & 0 .\end{array}$

$\begin{array}{lllllll}\mathrm{N} & \mathrm{N} 1 & 0.03425 & 0.01228 & 0.32497 & 0 .\end{array}$

$\begin{array}{llllllll}\text { C } & \text { C1 } & 0.22773 & 0.20083 & 0.40329 & 0 .\end{array}$

$\begin{array}{llllllll}\text { C } & \text { C2 } & 0.12204 & 0.01647 & 0.39089 & 0 .\end{array}$

C C3 $0.09716-0.169260 .440790$.

$\begin{array}{lllllll}\text { C } & \text { C } 4 & 0.17781 & -0.17376 & 0.50395 & 0 .\end{array}$

C C5 $0.28423 \quad 0.00540 \quad 0.51798 \quad 0$.

$\begin{array}{lllllllll}\text { C } & \text { C6 } & 0.30797 & 0.19008 & 0.46727 & 0 .\end{array}$

$\begin{array}{llllllll}\text { C C } & 0.37128 & 0.00175 & 0.58712 & 0 .\end{array}$

$\begin{array}{llllllll}\text { C } & \text { C8 } & 0.25417 & 0.40907 & 0.35124 & 0 .\end{array}$

$\begin{array}{lllllllll}\mathrm{H} & \mathrm{H} 2 & 0.38760 & 0.65111 & 0.32947 & 0 .\end{array}$

$\begin{array}{llllllll}\mathrm{H} & \mathrm{H} 3 & 0.01517 & -0.31080 & 0.43076 & 0 .\end{array}$

H H4 $0.15733-0.31896 \quad 0.54269 \quad 0$.

H $\quad \mathrm{H} 6 \quad 0.39072 \quad 0.32982 \quad 0.47734 \quad 0$.

H H1A $0.09223 \quad 0.01198 \quad 0.28473 \quad 0$.

H $\quad \mathrm{H} 1 \mathrm{~B} \quad-0.02454 \quad-0.15896 \quad 0.32245 \quad 0$.

$\mathrm{H} \quad \mathrm{H} 1 \mathrm{C} \quad-0.02642 \quad 0.18091 \quad 0.32153 \quad 0$.

H H7A $0.31820 \quad 0.09034 \quad 0.62504 \quad 0$.

H $\quad$ H7B $\quad 0.39595 \quad-0.19986 \quad 0.60110 \quad 0$.

H $\quad \mathrm{H} 7 \mathrm{C} \quad 0.46024 \quad 0.11225 \quad 0.58333 \quad 0$.

O OW $0.42920 \quad 0.67298 \quad 0.76040 \quad 0$.

$\begin{array}{lllllll}\mathrm{H} & \mathrm{H} 1 \mathrm{~W} & 0.48164 & 0.50919 & 0.76268 & 0 .\end{array}$

$\begin{array}{lllllll}\mathrm{H} & \mathrm{H} 2 \mathrm{~W} & 0.34648 & 0.62584 & 0.73260 & 0 .\end{array}$

Q Q01C8 $0.22014 \quad 0.43465 \quad 0.33024 \quad 0$.

Q $\quad$ Q02C8 $0.30466 \quad 0.45790 \quad 0.35727 \quad 0$.

$\mathrm{Q} \quad \mathrm{QO} 2 \mathrm{H} 20.383650 .61552 \quad 0.339590$.

Q QN1C2 $0.07290 \quad 0.01413 \quad 0.353990$.

Q $\mathrm{Q} 5-0.00138-0.09151 \quad 0.32344 \quad 0$.

$\mathrm{Q} \quad \mathrm{Q} 6-0.00197 \quad 0.11295 \quad 0.322910$.

Q $\quad 27 \quad 0.06982 \quad 0.01210 \quad 0.30028 \quad 0$.

Q QC1C8 $0.24276 \quad 0.319190 .37371 \quad 0$.

Q $\quad$ QC1C2 $0.17167 \quad 0.10304 \quad 0.39671 \quad 0$.

Q $\quad$ QC1C6 $0.26934 \quad 0.19526 \quad 0.43647 \quad 0$.

$\mathrm{Q} \quad \mathrm{QC2C3} \quad 0.11008-0.07284 \quad 0.41488 \quad 0$.

$\begin{array}{llllllll}\mathrm{Q} & \mathrm{QC} 3 \mathrm{C} 4 & 0.13791 & -0.17154 & 0.47270 & 0 .\end{array}$

$\mathrm{Q} \quad \mathrm{QC} 3 \mathrm{H} 3 \quad 0.04479-0.25967 \quad 0.43438 \quad 0$.

$\mathrm{Q}$ QC4C5 $0.23062-0.08486 \quad 0.510910$.

Q QC4H4 $0.16510 \quad-0.26384 \quad 0.52798 \quad 0$.

Q $\quad$ QC5C6 $0.29630 \quad 0.09926 \quad 0.49221 \quad 0$.

$\begin{array}{llllllll}\mathrm{Q} & \mathrm{QC5C7} & 0.32689 & 0.00361 & 0.55186 & 0 .\end{array}$

Q $\quad$ QC6H6 $0.35976 \quad 0.27753 \quad 0.47357 \quad 0$.

Q $\quad \mathrm{Q} 19 \quad 0.42842 \quad 0.07272 \quad 0.58469 \quad 0$.

$\begin{array}{llllllll}\mathrm{Q} & \mathrm{Q} 20 & 0.38680 & -0.12507 & 0.59591 & 0 .\end{array}$

$\mathrm{Q} \quad \mathrm{Q} 210.338110 .05712 \quad 0.61082 \quad 0$.

$\mathrm{Q} \quad \mathrm{Q} 220.47033 \quad 0.54453 \quad 0.76218 \quad 0$.

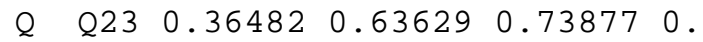

$\begin{array}{lllllll}\mathrm{Q} & \text { LP1 } & 0.18338 & 0.50772 & 0.29455 & 0 .\end{array}$

Q LP2 $0.15143 \quad 0.43792 \quad 0.301860$.

$\begin{array}{llllllll}\mathrm{Q} & \text { LP3 } & 0.39373 & 0.48213 & 0.36776 & 0 .\end{array}$

Q LP4 $\quad \begin{array}{llllll}37644 & 0.54653 & 0.37915 & 0 .\end{array}$

$\mathrm{Q} \quad \begin{array}{lllllll}\text { LP5 } & 0.44205 & 0.70647 & 0.75197 & 0 .\end{array}$

Q LP6 $0.42135 \quad 0.67782 \quad 0.773490$.

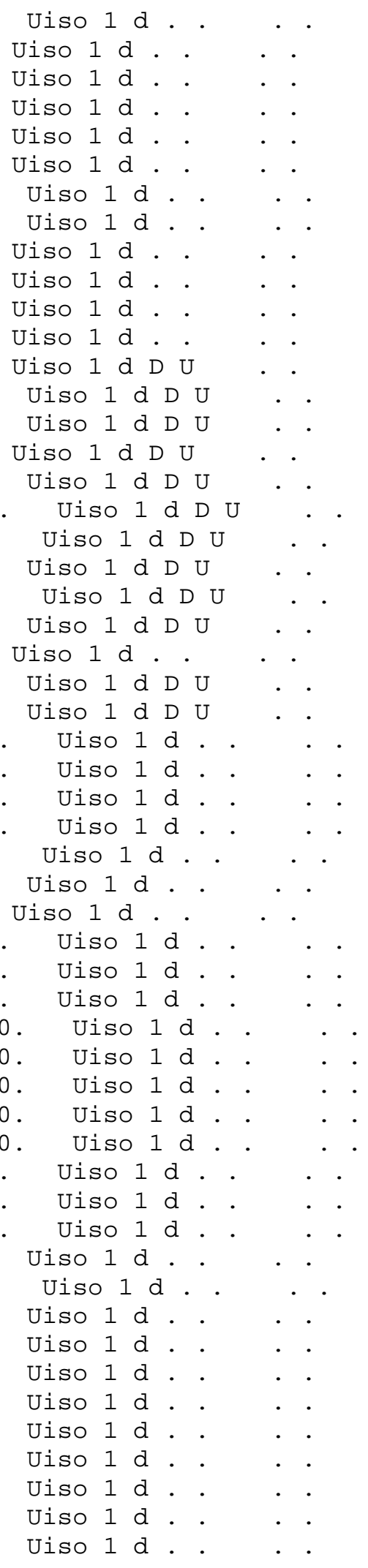

loop

_geom_hbond_atom_site_label_D

_geom_hbond_atom_site_label_H

_geom_hbond_atom_site_label_A 


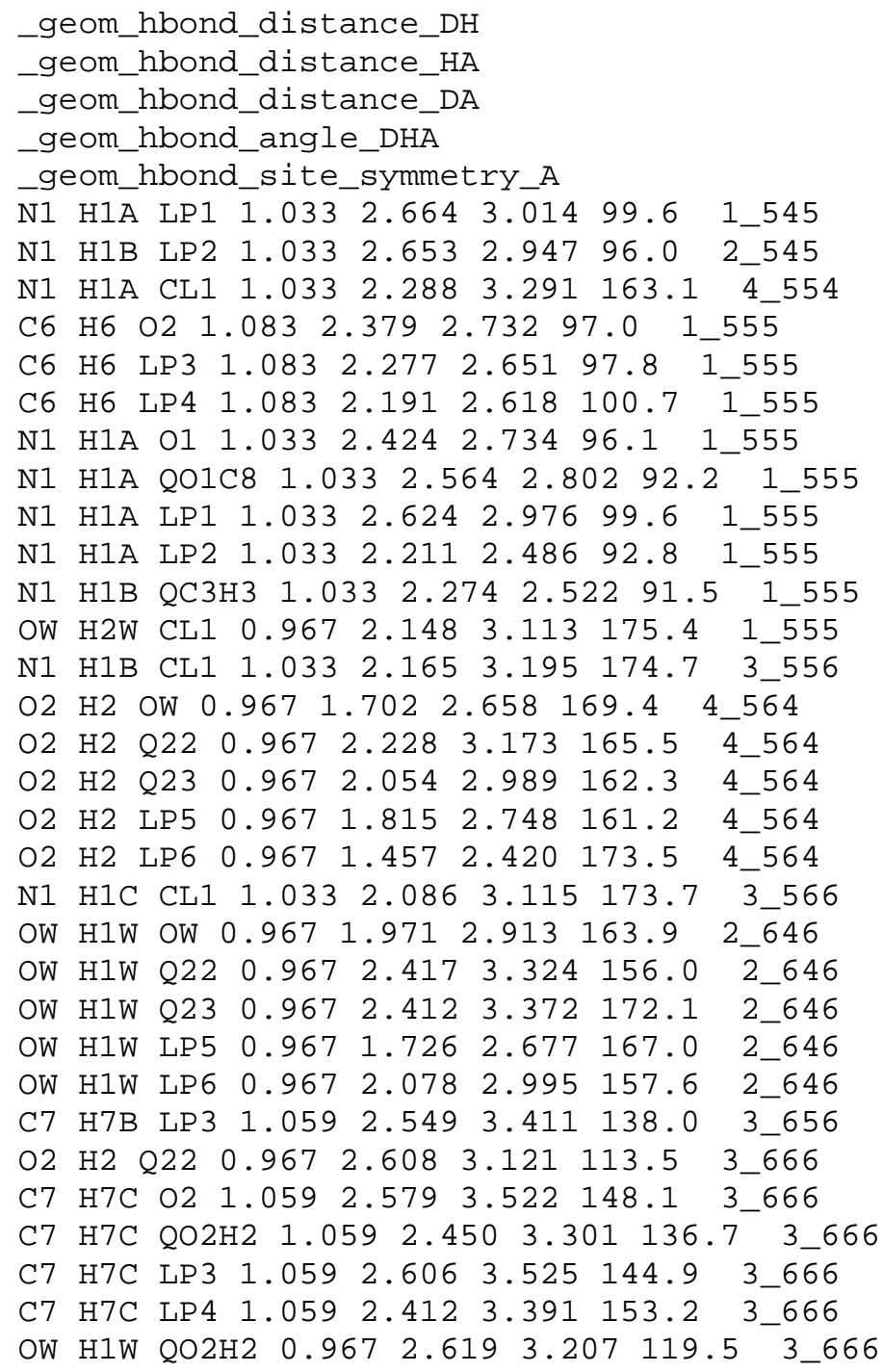

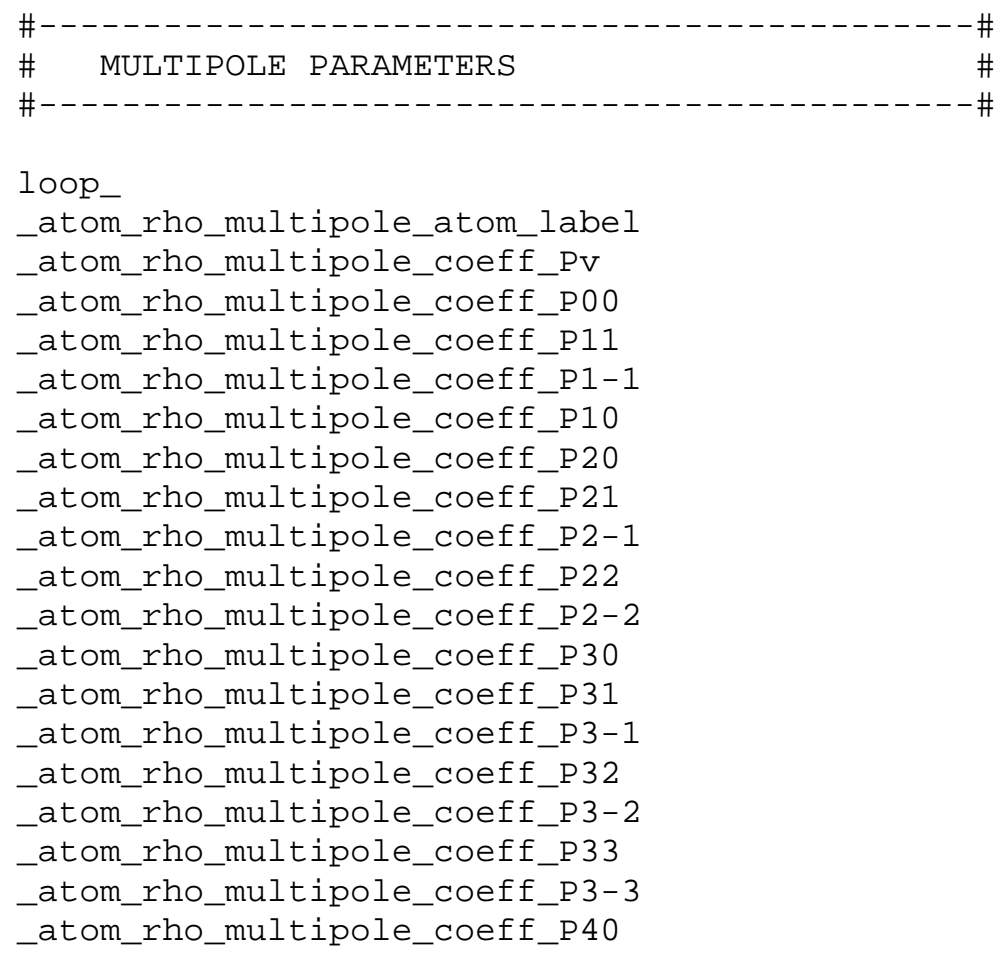




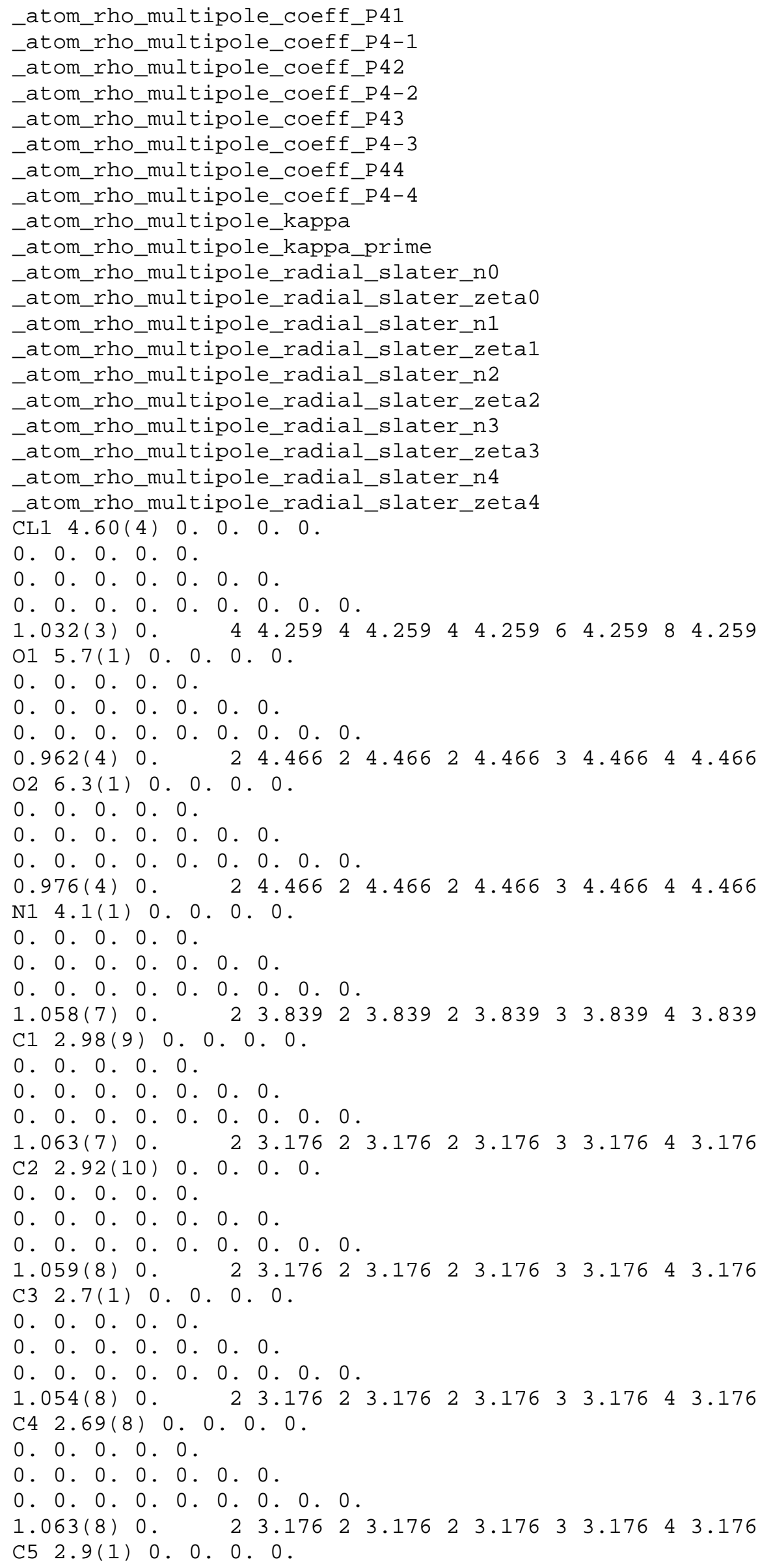




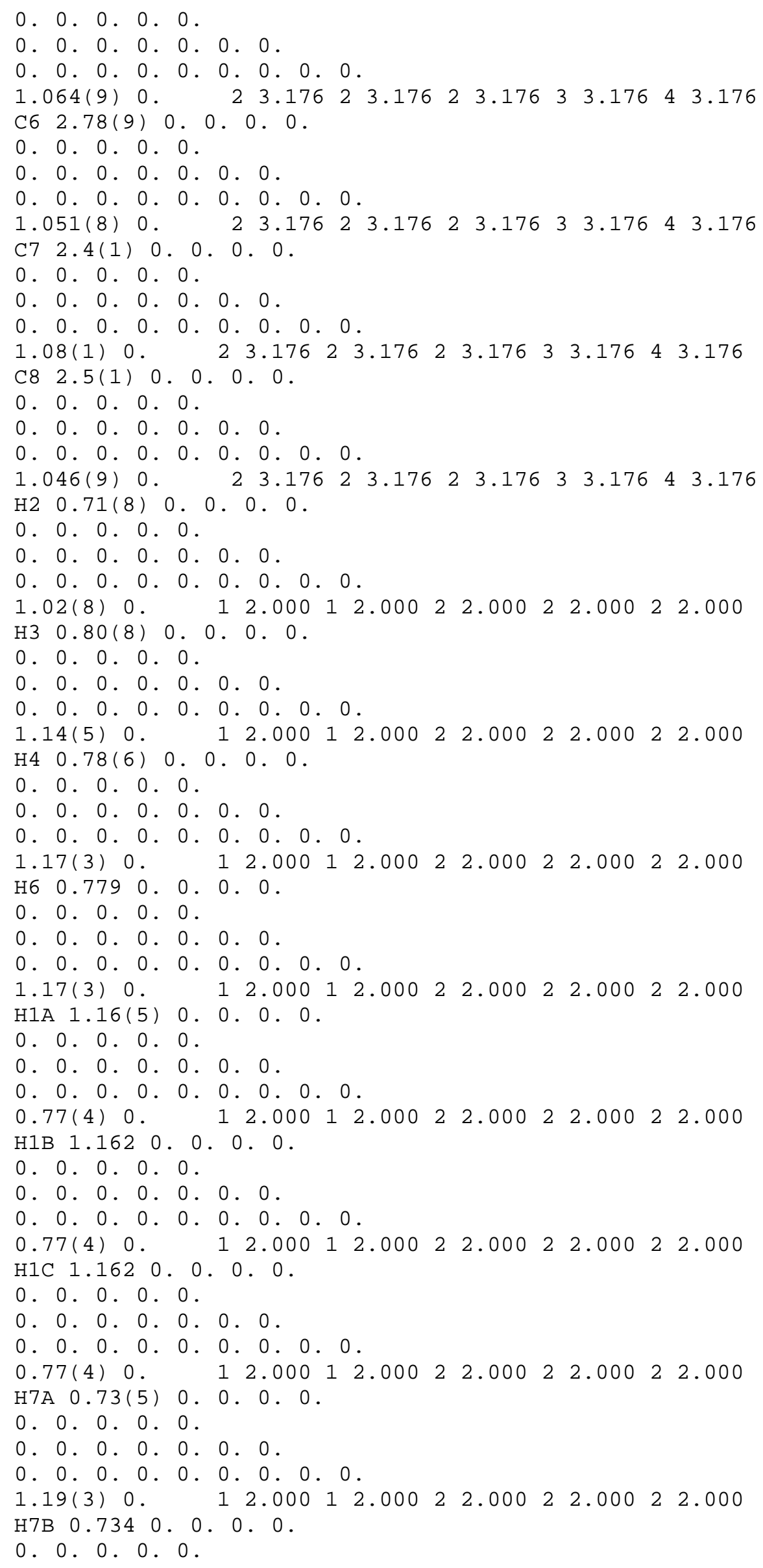




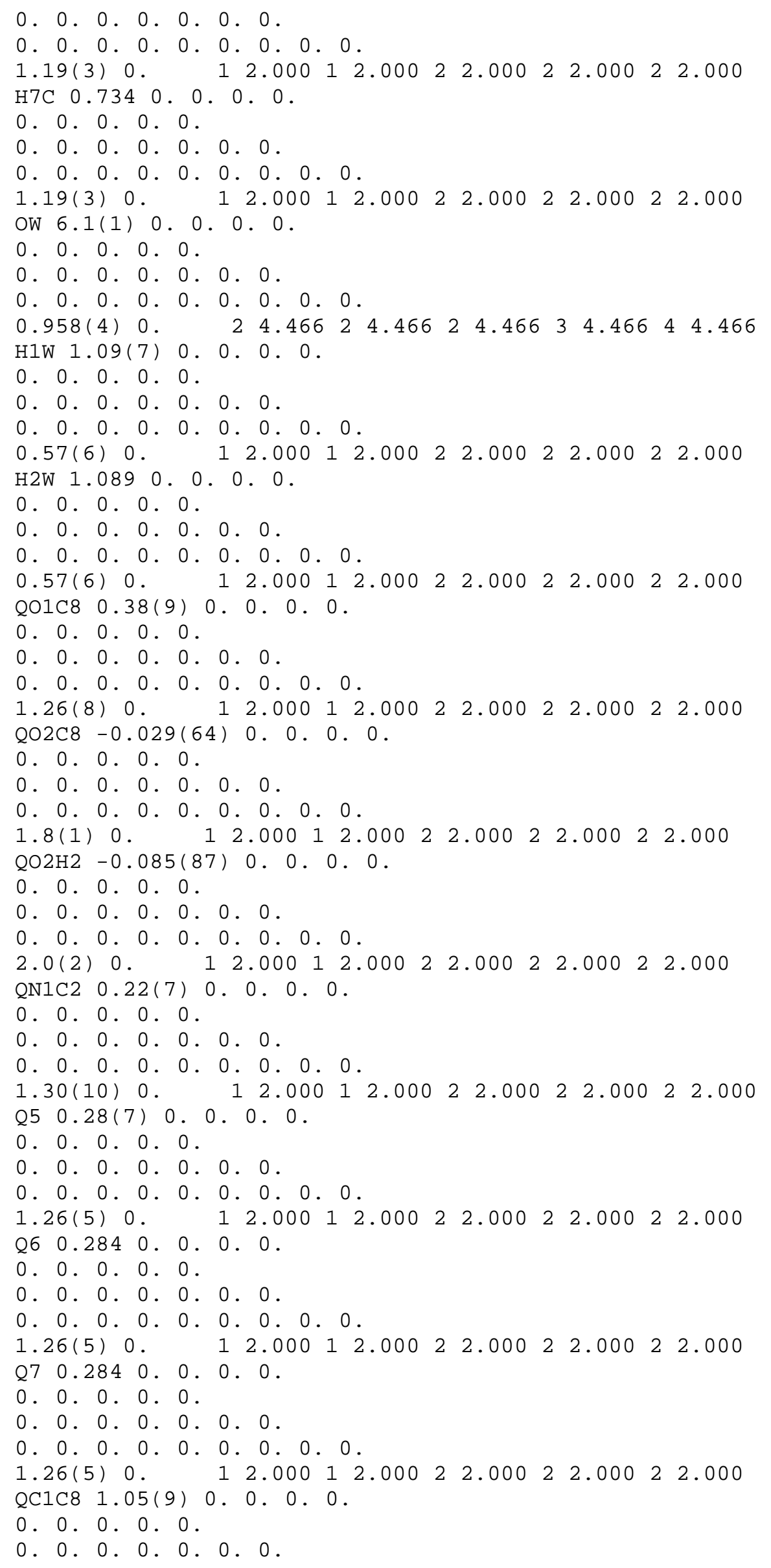




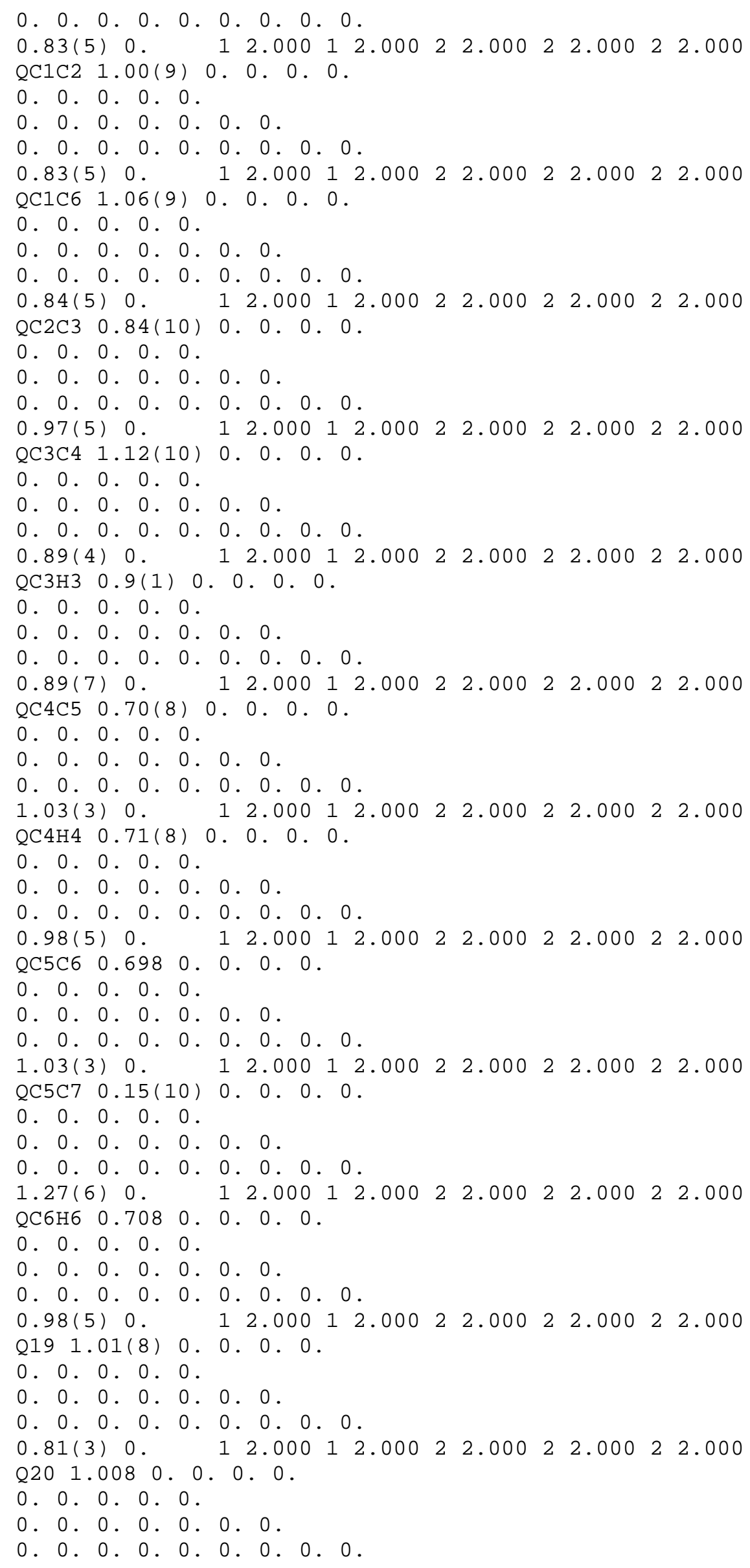


$0.81(3) \quad 0 . \quad 12.00012 .00022 .00022 .00022 .000$ Q21 1.008 0. 0. 0.0 .

0.0 .0 .0 .0 .

0.0 .0 .0 .0 .0 .0 .

0. 0.0.0.0.0.0.0.0. 0.

$0.81(3) 0 . \quad 12.00012 .00022 .00022 .00022 .000$

Q22 $0.012(70) 0.0 .0 .0$.

0.0 .0 .0 .0 .

0.0 .0 .0 .0 .0 .0 .

0.0.0.0.0.0.0.0.0.0.

$2.0(1) \quad 0 . \quad 12.00012 .000222 .00022 .00022 .000$ Q23 0.0120 .0 .0 .0 .

0.0 .0 .0 .0 .

0.0 .0 .0 .0 .0 .0 .

0.0 .0 .0 .0 .0 .0 .0 .0 .

$2.0(1) 0 . \quad 12.00012 .00022 .000222 .00022 .000$

LP $10.25(5) \quad 0.0 .0 .0$.

0.0 .0 .0 .0 .

0.0 .0 .0 .0 .0 .0 .

0.0.0.0.0.0.0.0.0.0.

$1.7(1) 0 . \quad 12.00012 .00022 .000222 .00022 .000$

LP2 0.2550 .0 .0 .0 .

0.0 .0 .0 .0 .

0.0 .0 .0 .0 .0 .0 .

0.0 .0 .0 .0 .0 .0 .0 .0 .

$1.7(1) \quad 0 . \quad 12.00012 .00022 .00022 .00022 .000$ LP3 $0.06(3) 0.0 .0 .0$.

0.0 .0 .0 .0 .

0.0 .0 .0 .0 .0 .0 .

0.0 .0 .0 .0 .0 .0 .0 .0

$2.3(2) 0 . \quad 12.00012 .00022 .000222 .00022 .000$ LP 40.0610 .0 .0 .0 .

0.0 .0 .0 .0 .

0.0 .0 .0 .0 .0 .0 .

0.0 .0 .0 .0 .0 .0 .0 .0 .

$2.3(2) \quad 0 . \quad 12.00012 .00022 .00022 .00022 .000$ LP $50.19(4) 0.0 .0 .0$.

0.0 .0 .0 .0 .

0.0 .0 .0 .0 .0 .0 .

0.0 .0 .0 .0 .0 .0 .0 .0 .

$1.8(2) 0 . \quad 12.00012 .00022 .00022 .00022 .000$

LP 60.1950 .0 .0 .0 .

0.0 .0 .0 .0 .

0.0.0.0.0.0.0. 0 .

0.0 .0 .0 .0 .0 .0 .0 .0 .

$1.8(2) 0 . \quad 12.00012 .00022 .00022 .00022 .000$

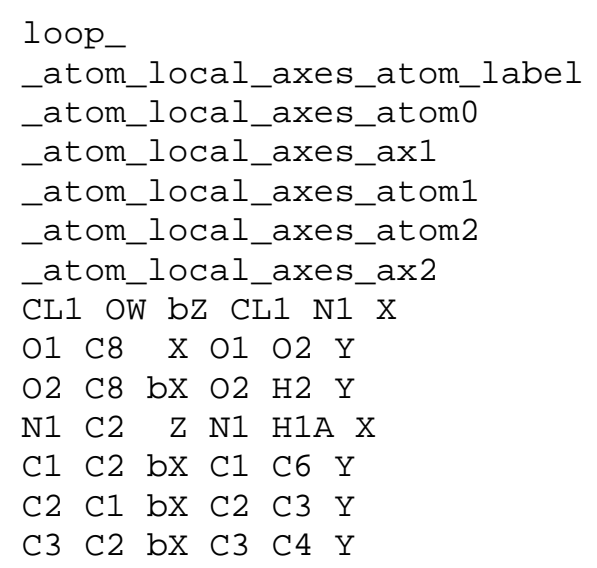




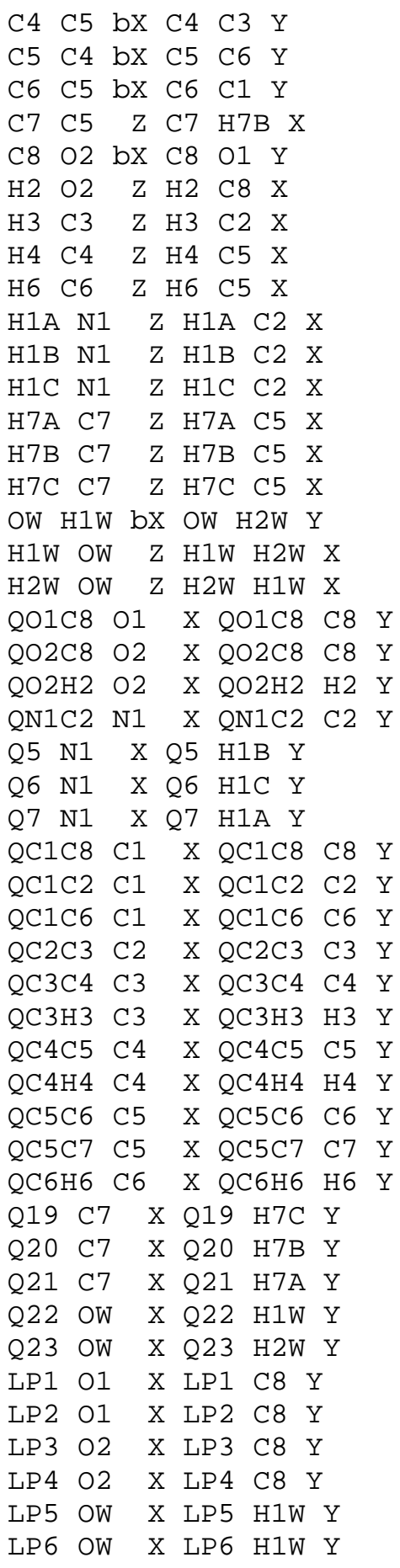

